\title{
Trauma epidemiology after easing of lockdown restrictions: experience from a level-one major trauma centre in England
}

\author{
Saima Waseem ${ }^{1}$ [ $\cdot$ Ramdeep Romann ${ }^{1} \cdot$ Jonathan Lenihan ${ }^{1} \cdot$ Jaikirty Rawal ${ }^{1} \cdot$ Andrew Carrothers $^{1} \cdot$ Peter Hull $^{1}$. \\ Daud Chou ${ }^{1}$
}

Received: 24 January 2021 / Accepted: 5 June 2021 / Published online: 25 June 2021

○) Springer-Verlag GmbH Germany, part of Springer Nature 2021

\begin{abstract}
Purpose The COVID-19 pandemic transformed the delivery of trauma care. We examined the effect of lockdown easing on trauma presentation and management from one Major Trauma Centre (MTC).

Methods Data was retrospectively analysed from Trauma Audit and Research Network (TARN) on patients presenting to our MTC with trauma. The first 47 days of lockdown (23rd March-9th May 2020, period 1) were compared with the next 47 (10th May-26th June 2020, period 2) and last (27th June-13th August 2020, period 3). Data collected included demographics, mechanism and severity of injury, management and length of stay.

Results 1249 patients were included; 62.2\% were male with a mean age of 57.73. Footfall declined in April 2020 compared with 2019 (56 vs. 143) but rebounded by May (123 vs. 120 patients). Road traffic collisions increased over periods one-three $(18.8 \%$ vs. $23 \%$ vs. $30.1 \%, p=0.038)$; deliberate self-harm $(\mathrm{DSH})$ increased in period two compared with one and three ( $6.3 \%$ vs. $3.4 \%$ vs. $1.4 \%, p=0.03$ ), respectively. When compared with 2019 , the 2020 patient age was lower, with less trauma relating to alcohol $(7.3 \%$ vs. $13.2 \%, p=0.009)$, but more from DSH (3.6\% vs. $2.1 \%, p=0.10)$. In 2020 less patients were assessed by a consultant and trauma team, with a shorter stay in hospital and critical care.

Conclusion This is the first study to document trauma trends through a lockdown and thereafter. After lockdown easing, trauma footfall rapidly rebounded to 2019 levels. This should be acknowledged in resource allocation decisions if future lockdowns are necessitated.
\end{abstract}

Keywords Major trauma $\cdot$ Polytrauma $\cdot$ Lockdown $\cdot$ Pandemic $\cdot$ COVID-19

\section{Introduction}

The COVID-19 pandemic and subsequent national lockdowns transformed the practice of medicine and surgery, including the delivery of trauma care. Service provision of surgical specialties was restricted by the repurposing of theatres to inpatient beds for COVID-positive patients, redeployment of theatre staff and trainees and staff sickness [1]. Structural changes were in part directed by National Health Service England (NHSE), with further advice published by the British Orthopaedic Association (BOA) [2]. There have

Saima Waseem

saimawaseem@doctors.org.uk

1 Cambridge Orthopaedic Trauma Unit (COTU), Addenbrooke's Major Trauma Centre, Cambridge University Hospitals NHS Foundation Trust, Hills Road, Cambridge CB20QQ, UK been multiple centres across the United Kingdom (UK) that have documented the alterations in the presentation and management of trauma secondary to the COVID-19 pandemic. Reported reductions in trauma presentation in the UK have ranged from 34 to $61 \%$ [3-5], with an unsurprising increase in incidents occurring at home with a concomitant reduction in road traffic collisions (RTCs) $[3,4,6]$. The UK literature also alludes to more concerning effects of lockdown such as a rise in deliberate self-harm (DSH) $[4,6,7]$ and assault [4].

In the UK, the first easing of the national lockdown was announced on 10th May, as the public were allowed to exercise more than once a day and spend time in parks. From 1st June, primary schools were re-opened. On 4th July -which became known as 'Super Saturday'- public houses, restaurants and non-essential businesses were allowed to open [8]. With this sequential easing of lockdown, we would anticipate an increase in trauma. In parallel, many hospitals in the 
UK turned their attention to catching up on their elective waiting lists and therefore had started diverting resources away from trauma to elective surgery, with national targets for $70 \%$ elective surgery to resume in August, and increase to $100 \%$ by October 2020 [9].

This study aimed to ascertain the impact of the easing of lockdown on the presentation and management of traumatic injuries at a Level 1 Major Trauma Centre (MTC) in England. To our knowledge, there has been no other paper to discuss this inevitable change in trauma, or a paper that has characterised the management of trauma in hospitals returning to normal levels of elective surgery.

\section{Methods}

All patients presenting to a single MTC in the East of England with a TARN eligible traumatic injury between 23 March and 31st August were retrospectively analysed, comparing the footfall of trauma in the lockdown period (23rd March-9th May) with the easing of lockdown (10th May-31st August). To do this, we compared the first 47 days of lockdown (23rd March-9th May, period 1). During this period all residents were advised to stay at home apart from key workers, and exercise was allowed once a day. In the next 47 (10th May-26th June, period 2), primary schools were opened (June 1st), residents were allowed to exercise more than once a day and congregate outdoors in small groups. In the following 47 days (27th June-13th August, period 3), on 4th July -dubbed 'Super Saturday' public houses, restaurants and non-essential shops were allowed to open. Residents were allowed to congregate both indoors and outdoors, but masks and social distancing were still recommended.

Inclusion criteria were all patients with a traumatic injury to any part of the body with an injury severity score (ISS) over 8 , which satisfied the TARN criteria for trauma. Data was sourced from the TARN database, and supplemented by electronic patient records. There were no exclusion criteria. Collected data included patient demographics and mechanism of injury, ISS and presenting Glasgow coma score (GCS). In addition, intubation on arrival and the presence of haemodynamic shock (defined as a systolic blood pressure under $110 \mathrm{mmHg}$ ) was documented. Finally, data were collected on patient management, including the grade of the doctor assessing the patient in the emergency department, use of a trauma computed tomography (CT) scan and number of operative procedures performed. Data were collected on a database and securely stored and reported in accordance with the STROBE statement [10].

Statistical analysis was performed using SPSS (Version 27, IBM Corp). Chi-square was used for categorical variables, with Kruskal-Wallis and Mann-Whitney $U$ tests used for non-parametric data. Statistical significance was set at $p<0.05$.

\section{Results}

\section{Trauma footfall}

1,249 patients were included with a mean age of 57.73 years of age, with $62.2 \%$ being male. A summary of trauma footfall between April and August 2020 compared with the same time period in 2019 is shown in Fig. 1. Patient demographics are summarised in Table 1.

\section{Severity of trauma}

The severity of trauma during and after the national lockdown is summarised in Table 2. When compared to the same period in 2019, there was a reduced proportion of patients attending intubated $(16.2 \%$ vs. $12.2 \%, p=0.042)$, but no significant difference in the median presenting ISS $(p=0.602)$, presenting GCS $(p=0.717)$ and proportion presenting with shock $(p=0.130)$. There was a similar 30 -day mortality between these two groups ( $5.9 \%$ vs. $6.7 \%, p=0.571)$.

\section{Type of trauma}

The type of trauma is summarised in Table 3. There was a significant increase in road traffic collisions (RTCs) over the three periods, with a concomitant decrease in the incidence of high and low energy falls. Moreover, there was a significant increase in the proportion of injuries occurring secondary to deliberate self-harm (DSH) in Period 2, $(p=0.030)$. There was no statistical difference between incident location, or the most severely injured body part between the periods studied. In addition, there was no difference between trauma secondary to assault or alcohol use. When compared with the same period in 2019 , there was a significant reduction in trauma related to alcohol consumption during the lockdown period $(7.3 \%$ vs. $13.2 \%, p=0.009)$ but a rise in trauma secondary to DSH (3.6\% vs. $2.1 \%, p=0.100)$, which was just subthreshold for statistical significance. There was no significant difference in the prevalence of assault during lockdown when compared to 2019 ( $4.2 \%$ vs. $5.3 \%, p=0.343$ ).

\section{Management of trauma}

The management of trauma is summarised in Table 4. There was a significant increase in the number of patients having a trauma computed tomography (CT) scan performed in period 3, with an increased overall length of stay for patients in period 2 although not statistically significant $(p=0.080)$. There was a reduced time to theatre in periods 1 and 2 when 
188

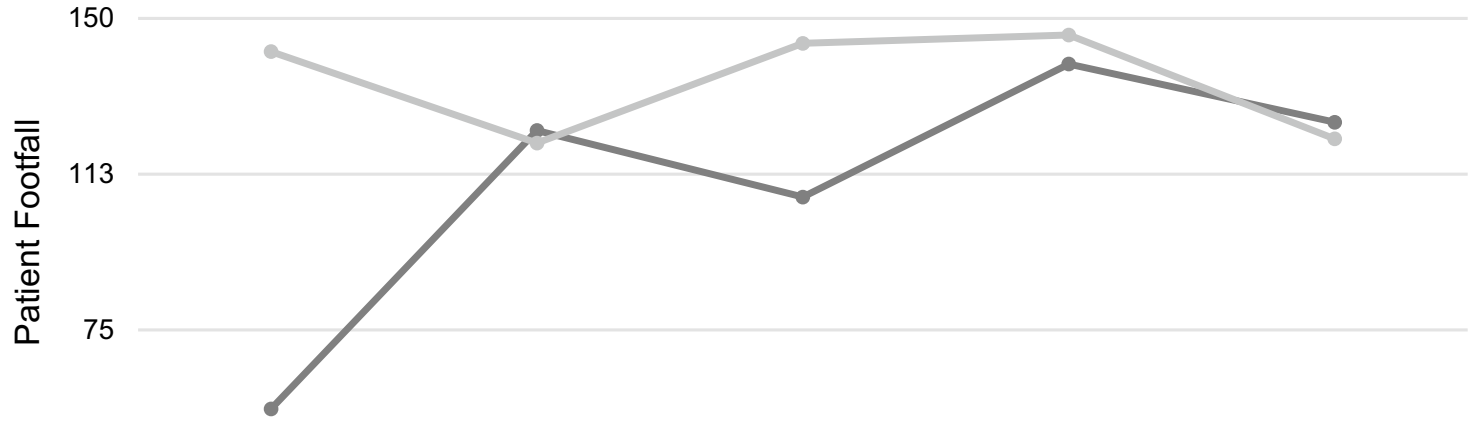

38

0

A

April May June $\quad$ July $\quad$ August

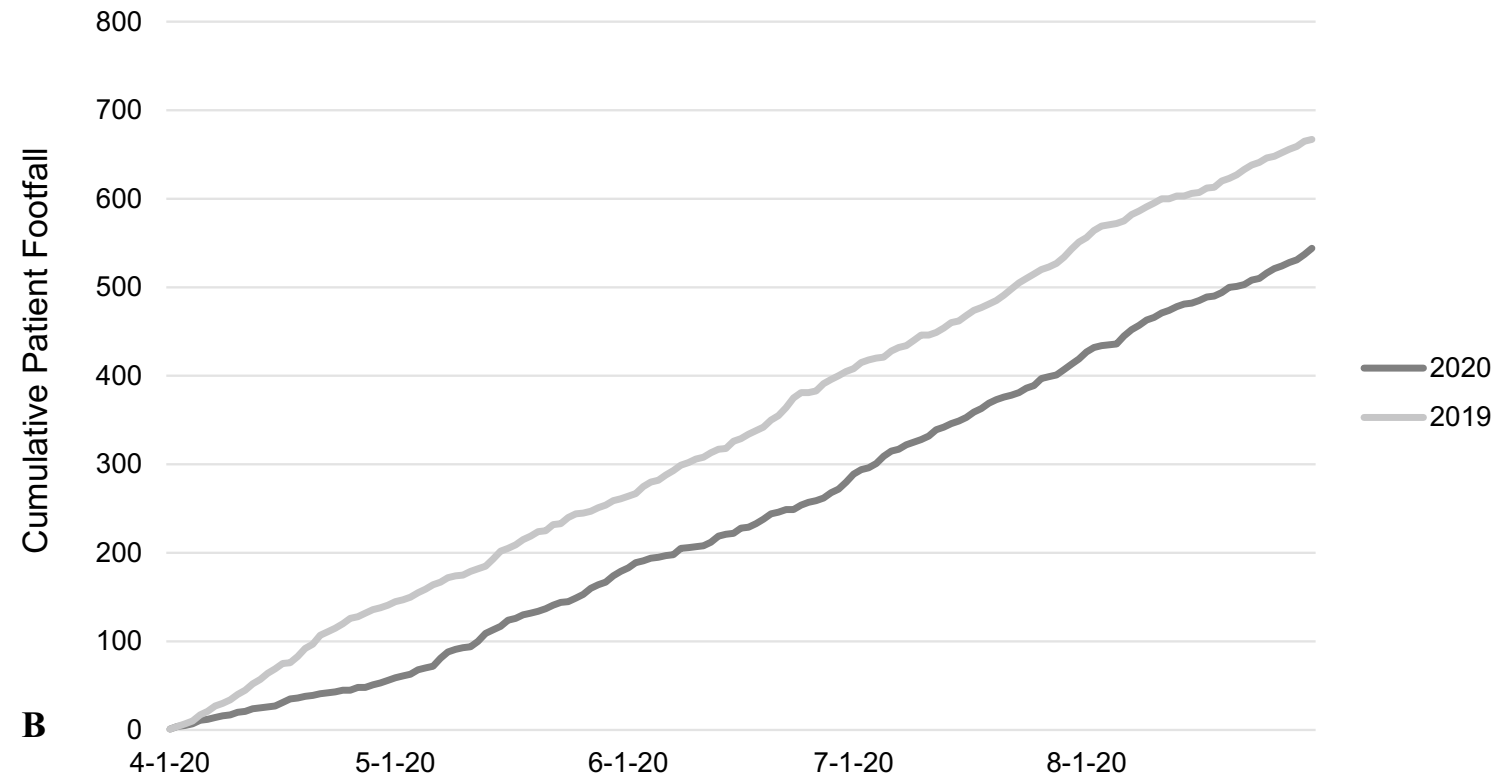

Fig. 1 Graphical Presentation of the epidemiology of trauma during the post-lockdown period, compared with 2019. Graphic description of trauma trends in the lockdown and post-lockdown period in 2020 ,

compared with period 3, however, this was below statistical significance.

When compared to 2019 , there was a significantly reduced mean total length of stay (14.10 vs. 17.35 days, $p \leq 0.001)$ and length of stay in critical care (2.26 vs. 2.86 days, $p=0.040$ ). Moreover, in 2019 a significantly compared with 2019. B Graphical description of the cumulative daily footfall of trauma patients presenting in the lockdown and post lockdown period compared with 2019

higher proportion of patients were assessed by a consultant (64.9\% vs. $54.9 \%, p<0.001)$. Overall, there was no significant difference between the proportion of patients requiring operative intervention between 2020 and 2019 (55.9\% vs. $61.0 \%, p=0.170)$. In addition, there was no significant 
Table 1 Patient demographics presenting with trauma during and after national lockdown

\begin{tabular}{lllll}
\hline & Period 1 & Period 2 & Period 3 & $p$ value \\
\hline$N$ & 117 & 174 & 219 & \\
Age (median) & 65.8 & 63.35 & 65.0 & 0.971 \\
Male (\%) & $73(62.4)$ & $107(61.5)$ & $140(63.9)$ & 0.881 \\
Transfer (\%) & $50(42.7)$ & $67(38.5)$ & $96(43.8)$ & 0.551 \\
Mode of arrival (\%) & & & & \\
Ambulance & $55(47.0)$ & $90(51.7)$ & $102(46.6)$ & 0.738 \\
Helicopter/Ambulance & $3(2.6)$ & $10(5.7)$ & $8(3.7)$ & \\
Car & $7(6.0)$ & $8(4.6)$ & $17(7.8)$ & \\
Helicopter & $9(7.7)$ & $9(5.2)$ & $14(6.4)$ & \\
Unknown & $43(36.8)$ & $57(32.8)$ & $78(3.6)$ & \\
\hline
\end{tabular}

Table 2 Severity of presenting trauma during and after lockdown

\begin{tabular}{lllll}
\hline & Period 1 & Period 2 & Period 3 & $p$ \\
\hline ISS, median & 16 & 17 & 16 & 0.961 \\
GCS, median & 15 & 15 & 15 & 0.349 \\
Shock (\%) & $27(23.1)$ & $29(16.7)$ & $32(14.6)$ & 0.143 \\
Intubated (\%) & $14(12.0)$ & $25(14.4)$ & $28(12.8)$ & 0.821 \\
Glasgow Outcome Score (\%) & & & \\
1 & $8(6.8)$ & $11(6.3)$ & $13(5.9)$ & 0.065 \\
2 & 0 & $2(1.1)$ & 0 & \\
3 & $4(3.4)$ & $8(4.6)$ & $4(1.8)$ & \\
4 & $29(24.8)$ & $55(31.6)$ & $44(20.1)$ & \\
5 & $76(65.0)$ & $97(55.7)$ & $158(72.1)$ & \\
Unknown & 0 & $1(0.6)$ & 0 & \\
Most severely injured body part & & & \\
Abdomen & $3(2.6)$ & $7(4.0)$ & $10(4.7)$ & 0.795 \\
Chest & $16(13.7)$ & $24(13.8)$ & $41(18.7)$ & \\
Face & $1(0.9)$ & $2(1.1)$ & 0 & \\
Head & $35(29.9)$ & $54(31.0)$ & $61(28.2)$ & \\
Limbs & $38(32.5)$ & $46(26.4)$ & $61(28.2)$ & \\
Multiple & $14(12.0)$ & $18(10.3)$ & $20(9.1)$ & \\
Spine & $10(8.5)$ & $21(12.1)$ & $25(11.4)$ & \\
Other & 0 & $2(1.1)$ & $1(0.5)$ & \\
30-day mortality (\%) & $9(7.7)$ & $11(6.3)$ & $12(5.5)$ & 0.728 \\
\hline
\end{tabular}

difference in a delay to the theatre between lockdown when compared with 2019 (2988 min vs. 2739 min, $p=0.560$ ).

\section{Changes in trauma after the opening of primary schools}

Changes in the management and presentation of trauma after the opening of primary schools on June 1st-31st August 2020 when compared with the same period in 2019 is summarised in Table 5. Compared with 2019, the presenting age of the patient was older (66.2 vs. 58.8 years, $p=0.046$ ), with a lower proportion intubated $(p=0.031)$ with less assessed with a trauma team present $(p=0.013)$. There was a lower proportion of patients sustaining trauma secondary to alcohol consumption during 2020 when compared with 2019 $(p=0.051)$. Finally, the patient's length of stay in hospital and in critical care was significantly lower.

\section{Changes in trauma after 'Super Saturday'}

The changes in trauma following the re-opening of restaurants, public houses and non-essential shops is shown in Table 6. The findings were similar to those after June 2020, with a significantly lower length of hospital stay, and less people arriving intubated. The mechanism of injury and trauma management was largely comparable between 2019 and 2020 .

\section{Discussion}

This study shows that after the first steps towards the easing of lockdown in May 2020, the footfall of trauma patients rebounded towards pre-lockdown levels. As the lockdown was gradually lifted, the presentation of trauma resembled 2019-with a higher proportion of injuries occurring secondary to RTCs. However, during and after the lockdown period there remained a reduction in patients being assessed by a consultant on arrival to the emergency department and a significantly reduced length of stay when compared with 2019.

The rise in footfall of trauma patients after the first easing of lockdown could reflect an increase in the movement of patients outside their homes, sustaining trauma when doing so. This is particularly shown by the rise in RTCs throughout the post-lockdown period. Absolute reductions in trauma secondary to RTCs during lockdown has been shown in multiple centres across the United Kingdom, including London $[4,11]$ and the North West of England [6]. Our data shows that in the months after lockdown (period 1), this reduction was being reversed. Further research is required to ascertain the trend in trauma in the longer term after lockdown (including following the second wave) to ascertain if trauma volume rises above 2019 levels as the lockdown eases.

Our results show a striking trend in the rates of trauma secondary to DSH and alcohol abuse during and after lockdown. Our data has shown a significant rise in traumatic injuries secondary to DSH during the first easing of lockdown, with higher rates of DSH compared to 2019 (although just beneath statistical significance). This observed increase is resolved as more restrictions are eased, for example from 'Super Saturday'. These results are an avid reflection of the detrimental effect of national lockdown on the mental health of the public and suggest that healthcare providers must be mindful of the negative 
Table 3 Types of Presenting trauma during and following the easing of lockdown

\begin{tabular}{lllll}
\hline & Period 1 & Period 2 & Period 3 & $p$ value \\
\hline Injury mechanism (\%) & & & & \\
Blows & $3(2.6)$ & 0 & 0 & $\mathbf{0 . 0 3 8}$ \\
Blunt trauma with weapon & 0 & 0 & 0 & \\
Crush & 0 & $1(0.6)$ & $1(0.5)$ & \\
Fall $<2 \mathrm{~m}$ & $69(59.0)$ & $96(55.2)$ & $112(51.9)$ & \\
Fall $>2 \mathrm{~m}$ & $17(14.5)$ & $20(11.5)$ & $20(9.1)$ & \\
Other & $1(0.9)$ & $7(4.0)$ & $4(1.8)$ & \\
Stabbing & $3(2.6)$ & $3(1.7)$ & $2(0.9)$ & \\
RTC & $22(18.8)$ & $40(23.0)$ & $66(30.1)$ & 0.769 \\
Deliberate self-harm (DSH) $(\%)$ & $4(3.4)$ & $11(6.3)$ & $3(1.4)$ & 0.569 \\
Alcohol related trauma (\%) & $10(8.5)$ & $15(8.6)$ & $15(2.3)$ & \\
Assault (\%) & $7(6.0)$ & $6(3.4)$ & $9(4.1)$ & \\
Location of injury (\%) & & & & \\
Garden & $5(4.3)$ & $6(3.4)$ & $14(6.4)$ & \\
Home & $59(50.4)$ & $74(42.5)$ & $77(35.2)$ & \\
Indoors & 0 & $1(0.6)$ & $2(0.9)$ & $116(53.0)$ \\
Outdoors & $47(40.2)$ & $87(50.0)$ & $10(4.6)$ & \\
Unknown & $6(5.1)$ & $6(3.4)$ &
\end{tabular}

\begin{tabular}{llllr}
\hline & Period 1 & Period 2 & Period 3 & $p$ value \\
\hline Trauma team present (\%) & 117 & 174 & 219 & \\
Most senior Assessing in ED (\%) & $24(20.5)$ & $38(21.8)$ & $50(22.8)$ & 0.886 \\
Consultant & & & & \\
ST3+ & $58(49.6)$ & $100(57.5)$ & $126(57.5)$ & 0.107 \\
Junior & $32(27.4)$ & $46(26.4)$ & $57(26.0)$ & \\
Other & $5(4.3)$ & $3(1.7)$ & $6(2.7)$ & \\
Unknown & $3(2.6)$ & 0 & 0 & \\
Trauma CT performed (\%) & $19(16.2)$ & $25(14.4)$ & $30(13.7)$ & \\
Median time to trauma CT (mins) & $29(24.8)$ & $19(10.9)$ & $70(32.0)$ & $<\mathbf{0 . 0 0 1}$ \\
Number requiring operative intervention (\%) & $74(63.2)$ & $89(51.1)$ & $122(55.7)$ & 0.125 \\
Median time to operation (mins) & 991 & 1283 & 1458 & 0.070 \\
Number of operations (median) & 1 & 1 & 1 & 0.768 \\
Length of stay, (mean days) & 13.38 & 16.65 & 13.21 & 0.076 \\
ICU length of stay (mean days) & 2.08 & 2.87 & 2.24 & 0.523 \\
\hline
\end{tabular}

Table 4 Management of trauma during and after national lockdown effects of lockdown and subsequent shift in aetiology of trauma that may present secondary to this. The detrimental impact of lockdown on mental health is well documented, including the effects of social isolation [12], and rise in DSH during lockdown periods [13]. In parallel, there has been a reported increase in some cases of assault [14], stabbing [7] and domestic violence [15], which was not observed in our study. This study suggests a relationship between the degree of restrictions placed on public free movement and footfall of traumatic injuries secondary to DSH. This is an important consideration when making a decision to implement further national lockdowns, and considering funding towards mental health services to meet demand.

Furthermore, this study has shown that the national lockdown initially decreased alcohol-related trauma. There is a well-described increased in alcohol consumption during lockdown [16, 17] with concomitant reported increases in alcohol-related trauma [14]. However, in our population a reduction in alcohol-related trauma was noted during the lockdown, and immediately after. This effect was reversed from 'Super Saturday', when alcohol-related trauma trended towards 2019 levels. This study has shown lockdown was associated with a reduction in alcohol-related trauma. This 
Table 5 Presentation and management of trauma between June 1st and August 31st 2020, compared with the same period in 2019

\begin{tabular}{|c|c|c|c|}
\hline & 2020 & 2019 & $p$ \\
\hline$N$ & 371 & 411 & \\
\hline Age (median) & 66.2 & 58.8 & 0.046 \\
\hline Male (\%) & $232(62.5)$ & $259(63.0)$ & 0.889 \\
\hline ISS (median) & 16 & 16 & 0.496 \\
\hline GCS (median) & 15 & 15 & 0.363 \\
\hline \multicolumn{4}{|l|}{ Mechanism (\%) } \\
\hline Blow with weapon & $1(0.3)$ & 0 & 0.540 \\
\hline Blow without weapon & $21(5.7)$ & $25(6.1)$ & \\
\hline Crush & $3(0.8)$ & $3(0.7)$ & \\
\hline Fall $<2 \mathrm{~m}$ & $195(52.6)$ & $195(47.4)$ & \\
\hline Fall $>2 \mathrm{~m}$ & $34(9.2)$ & $40(9.7)$ & \\
\hline Other & $8(2.2)$ & $8(1.9)$ & \\
\hline Shooting & 0 & $3(0.7)$ & \\
\hline Stabbing & $8(2.2)$ & $6(1.5)$ & \\
\hline RTC & $101(27.2)$ & $131(31.9)$ & \\
\hline Assault (\%) & $13(3.5)$ & $17(4.1)$ & 0.646 \\
\hline $\mathrm{DSH}(\%)$ & $12(3.2)$ & $11(2.7)$ & 0.645 \\
\hline Related to alcohol (\%) & $25(6.7)$ & $44(10.7)$ & 0.051 \\
\hline Transfer? $(\%)$ & $159(42.9)$ & $186(45.3)$ & 0.500 \\
\hline Intubated? (\%) & 43 (11.6) & $70(17.0)$ & 0.031 \\
\hline Shock? (\%) & $54(14.6)$ & $66(16.1)$ & 0.256 \\
\hline Trauma Team Present? (\%) & $82(22.1)$ & $123(29.9)$ & 0.013 \\
\hline \multicolumn{4}{|l|}{ Most Senior ED Doctor (\%) } \\
\hline Cons & $212(57.1)$ & $253(61.6)$ & 0.296 \\
\hline ST3+ & $89(24.0)$ & $76(18.5)$ & \\
\hline Junior (FY/ST1/2) & $10(2.7)$ & $10(2.4)$ & \\
\hline Unknown & $60(16.2)$ & $72(17.5)$ & \\
\hline Operative intervention? (\%) & $199(53.6)$ & $246(59.9)$ & 0.080 \\
\hline LOS (mean) & 13.25 & 17.76 & $<0.001$ \\
\hline ICU LOS (mean) & 2.13 & 2.92 & 0.003 \\
\hline 30-day mortality (\%) & $23(6.2)$ & $29(7.1)$ & 0.631 \\
\hline \multicolumn{4}{|l|}{ Glasgow Outcome Score (\%) } \\
\hline 1 & $24(6.5)$ & $28(6.8)$ & 0.098 \\
\hline 2 & 0 & $1(0.2)$ & \\
\hline 3 & $8(2.2)$ & $5(1.2)$ & \\
\hline 4 & $82(22.1)$ & $122(29.7)$ & \\
\hline 5 & $257(69.3)$ & $255(62.0)$ & \\
\hline
\end{tabular}

may suggest that although increased alcohol consumption at home has been widely reported [16, 17], this has not translated into a higher proportion of alcohol-related trauma.

Further justification for the rebound of trauma footfall could include reduced public compliance with lockdown procedures after the lockdown was eased (periods 2 and 3). Public compliance with lockdown restrictions has been reported to be variable, with some reports of public compliance of $31 \%$ [18, 19]. Little is known about the rate of public compliance with increased duration of lockdown;
Table 6 Presentation and management of trauma between July 4th and August 31st 2020, compared with the same period in 2019

\begin{tabular}{|c|c|c|c|}
\hline & 2020 & 2019 & $p$ \\
\hline$N$ & 248 & 254 & \\
\hline Age (median) & 65.1 & 59.3 & 0.146 \\
\hline Male (\%) & $155(62.5)$ & $155(61.0)$ & 0.734 \\
\hline ISS (median) & 16 & 16 & 0.236 \\
\hline GCS (median) & 15 & 15 & 0.490 \\
\hline \multicolumn{4}{|l|}{ Mechanism } \\
\hline Blow with weapon & $1(0.4)$ & 0 & 0.288 \\
\hline Blow without weapon & $17(6.9)$ & $11(4.3)$ & \\
\hline Crush & $1(0.4)$ & $2(0.8)$ & \\
\hline Fall $<2 \mathrm{~m}$ & $130(52.4)$ & $118(46.5)$ & \\
\hline Fall $>2 \mathrm{~m}$ & $20(8.1)$ & $26(10.2)$ & \\
\hline Other & $3(1.2)$ & $5(2.0)$ & \\
\hline Shooting & 0 & $1(0.4)$ & \\
\hline Stabbing & $6(2.4)$ & $2(0.8)$ & \\
\hline RTC & $70(28.2)$ & $89(35.0)$ & \\
\hline DSH (\%) & $4(1.6)$ & $8(3.1)$ & 0.260 \\
\hline Assault (\%) & $5(2.0)$ & $9(3.5)$ & 0.259 \\
\hline Trauma related to Alcohol (\%) & $15(6.0)$ & $22(8.7)$ & 0.263 \\
\hline Transfer? (\%) & $112(45.2)$ & $104(40.9)$ & 0.340 \\
\hline Intubated? (\%) & $24(9.7)$ & $43(16.9)$ & 0.017 \\
\hline Shock? (\%) & $35(14.1)$ & $45(17.7)$ & 0.414 \\
\hline Trauma Team Present? (\%) & $53(21.4)$ & $75(29.5)$ & 0.036 \\
\hline \multicolumn{4}{|l|}{ Most Senior ED Doctor } \\
\hline Cons & $134(54.0)$ & $156(61.4)$ & 0.415 \\
\hline ST3+ & $61(24.6)$ & $6(2.4)$ & \\
\hline Junior (FY/ST1/2) & $7(29.2)$ & $51(20.1)$ & \\
\hline Unknown & $46(18.5)$ & $41(16.1)$ & \\
\hline Operative intervention? (\%) & $137(55.2)$ & $155(61.0)$ & 0.207 \\
\hline LOS (mean) & 12.87 & 17.83 & $<0.001$ \\
\hline ICU LOS (mean) & 1.91 & 2.92 & 0.001 \\
\hline 30-day mortality (\%) & $13(5.2)$ & $17(6.7)$ & 0.493 \\
\hline \multicolumn{4}{|l|}{ Glasgow outcome score } \\
\hline 1 & $14(5.6)$ & $17(6.7)$ & 0.084 \\
\hline 2 & 0 & $1(0.4)$ & \\
\hline 3 & $3(1.2)$ & $4(1.6)$ & \\
\hline 4 & $48(19.4)$ & $73(28.7)$ & \\
\hline 5 & $18(7.3)$ & $15(5.9)$ & \\
\hline
\end{tabular}

and policymakers have referred to behavioural fatigue when deciding on the timing of national lockdowns, however, it does not have a recognised scientific evidence base [20]. The results of our study indicate a fatigue with lockdown restrictions causing reduced adherence, leading to the footfall of trauma rebounding towards pre-COVID levels.

In terms of patient management, it was noted that there was less consultant involvement in the care of trauma patients during the 2020 pandemic. This in part contradicts the guidance published by the BOA released in July 2020 
encouraging senior Orthopaedic review to minimise the length of stay [2]. Furthermore, less patients were assessed with a trauma team present on their arrival during the lockdown period. A potential explanation for this could be staff absence and sickness, or senior staff being redeployed to provide care for critically unwell COVID-19 positive patients. Although current evidence indicates a detriment to trainee's operative experience secondary to the COVID-19 pandemic $[1,21]$, our results indicate that there was more emergency department and surgical trainee exposure to patients sustaining acute trauma. Our study has shown the increased use of trauma CT scans during the pandemic, which is in line with the COVID-19 guidance given by the BOA for the initial management of trauma during the pandemic [2].

Lastly, this study has shown a reduction in ICU and overall length of stay during the COVID-19 pandemic, despite 2020 trauma patients being significantly older, with a similar severity of trauma. Our data showed that despite the shorter length of stay, the mortality of patients in 2020 was unchanged when compared with 2019. The shortened length of stay likely represents attempts by hospitals to improve the efficiency of care to meet the additional demands of critically unwell COVID patients. Although the precise mechanisms employed to reduce hospital stay were not studied, potential methods include a lower threshold for repatriation to trauma units, and for step-down from critical care. Our study has suggested length of stay was safely shortened without affected mortality in these patients, and further analysis into methods adopted may yield learning points to improve healthcare efficiency in the post-pandemic era.

Whilst we have established guidelines for major incidents within our department, there is no such guidance in place for pandemics or similar disasters. COVID-19 exposed this weakness. Guidance was forthcoming at intervals from the National Health Service England (NHSE) as well as advice from the BOA, such as Consultant of the Day; Early senior Orthopaedic review; appropriate Personal Protective Equipment (PPE) to be worn; regular assessments of resources; documenting that decisions were taken in light of the pandemic; but this was only once the pandemic had started. Clear advice to cease elective operating was given by NHS England but guidance on how trauma should be managed was less clear. We recommend a general consensus from the Orthopaedic community as to how cases, in general, should be managed in future should similar events occur.

In a post-lockdown health service, emphasis has been placed on the return of elective operating to pre-lockdown levels [9]; however, the findings of our study pose potential limitations of this. Our study shows a rebound effect of trauma footfall and presentation from May 2020, and further study is required to ascertain the trend of this through the winter months. It may be conceivable that the rebound effect may rise the footfall of trauma patients higher than 2019 levels. This would place pressure on Trauma and Orthopaedic teams to provide safe, timely and effective care for these patients whilst concomitantly compensating for delays to care for elective patients. It is also clear that COVID-19 has not completely disappeared, and theatre and personnel resources may still be restricted [22]. The information in this study could therefore predict obstacles in the resumption of elective practice whilst balancing trauma care in a post lockdown society.

This study is not without its limitations. It is limited by its retrospective design, small patient numbers and data sourced from a single centre- and the effects observed may be confounded by other factors which the authors have not identified. This effect of trauma must be considered against the population studied. Our level 1 major trauma centre serves trauma units from across the East of England, which had a relatively low prevalence of COVID-19 compared to the remainder of the United Kingdom [23]. This is due to the largely rural population within this region, and preponderance of rural landscapes [24]. For this reason, the results of this study may not be generalisable to the entire UK population, and a multicentre study is required to clarify if the effect is observed in other centres.

Despite this, this is the first study to document the trend in trauma as the United Kingdom recovers from its first national lockdown. The results can be used to inform decision-making about rationing of resources such as redeploying trainees and theatres in the event of further waves of the pandemic. Our study has shown that footfall of trauma has recovered to pre-COVID levels, and resources should be sufficiently distributed to adequately meet demand.

Acknowledgements None.

Funding None.

\section{Declarations}

Conflict of interest None.

Consent for publication All authors have consented to publication.

\section{References}

1. Orthopaedic Research Collaborative (East) Anglia. The response of Trauma \& Orthopaedic Departments to the first four weeks of lockdown for the COVID-19 pandemic - a trainee-led analysis of the East of England. The Surg: J Royal Colleges Surg Edinburgh Ireland. 2021;19(1):e14-e19.

2. British Orthopaedic Association. Management of patients with urgent orthopaedic conditions and trauma during the coronavirus pandemic. London: British Orthopaedic Association; 2020. 
3. Garude K, Natalwala I, Hughes B, West C, Bhat W. Patterns of adult and paediatric hand trauma during the COVID-19 lockdown. J Plast Reconstr Aesthet Surg. 2020;73(8):1575-1592.

4. Rinkoff S, Jemec B. Variation in volumes and characteristics of hand trauma patients seen during the early COVID-19 lockdown in a central London Plastic Surgery Unit. Br J Surg. 2020;107(12):e571-e572.

5. Greenhalgh M, Dupley L, Unsworth R, Boden R. Where did all the trauma go? A rapid review of the demands on orthopaedic services at a U.K. Major Trauma Centre during the COVID-19 pandemic. Int J Clin Pract. 2021;75(3):e13690.

6. Rajput K, Sud A, Rees M, Rutka O. Epidemiology of trauma presentations to a major trauma centre in the North West of England during the COVID-19 level 4 lockdown. Eur J Trauma Emergency Surg. 2021;47(3):631-636.

7. Olding J, Zisman S, Olding C, Fan K. Penetrating trauma during a global pandemic: Changing patterns in interpersonal violence, self-harm and domestic violence in the Covid-19 outbreak. Surgeon. 2021;19(1):e9-e13.

8. Evie A. COVID-19 Timeline 2020. https://bfpg.co.uk/2020/04/ covid-19-timeline/.

9. Stevens S PA. Third Phase Response to COVID-19 2020. https:// www.england.nhs.uk/coronavirus/wp-content/uploads/sites/52/ 2020/07/20200731-Phase-3-letter-final-1.pdf.

10. Von Elm E, Altman DG, Egger M, Pocock SJ, Gøtzsche PC, Vandenbroucke JP. The Strengthening the Reporting of Observational Studies in Epidemiology (STROBE) statement: guidelines for reporting observational studies. Ann Intern Med. 2007;147(8):573-7.

11. Park C, Sugand K, Nathwani D, Bhattacharya R, Sarraf KM. Impact of the COVID-19 pandemic on orthopedic trauma workload in a London level 1 trauma center: the "golden month". Acta Orthop. 2020;91(5):556-561

12. Ammar A, Mueller P, Trabelsi K, Chtourou H, Boukhris O, Masmoudi L, et al. Psychological consequences of COVID-19 home confinement: the ECLB-COVID19 multicenter study. PLoS ONE. 2020;15(11):e0240204.

13. Waseem S, Nayar SK, Hull P, Carrothers A, Rawal J, Chou $\mathrm{D}$, et al. The global burden of trauma during the COVID-19 pandemic: a scoping review. J Clin Orthopaedics Trauma. 2021;12(1):200-207.

14. Rhodes HX, Petersen K, Biswas S. Trauma trends during the initial peak of the COVID-19 pandemic in the midst of lockdown: experiences from a rural trauma center. Cureus. 2020;12(8):9811.
15. Rhodes HX, Petersen K, Lunsford L, Biswas S. COVID-19 resilience for survival: occurrence of domestic violence during lockdown at a rural American college of surgeons verified level one trauma center. Cureus. 2020;12(8):10059.

16. Finlay I, Gilmore I. Covid-19 and alcohol-a dangerous cocktail. BMJ. 2020;369:m1987.

17. The Lancet Gastroenterology. Drinking alone: COVID-19, lockdown, and alcohol-related harm. Lancet Gastroenterol Hepatol. 2020;5(7):625.

18. Norman P, Wilding S, Conner M. Reasoned action approach and compliance with recommended behaviours to prevent the transmission of the SARS-CoV-2 virus in the UK. Br J Health Psychol. 2020;25(4):1006-19.

19. Coroiu A, Moran C, Campbell T, Geller AC. Barriers and facilitators of adherence to social distancing recommendations during COVID-19 among a large international sample of adults. PLoS ONE. 2020;15(10):0239795.

20. Mahase E. Covid-19: was the decision to delay the UK's lockdown over fears of "behavioural fatigue" based on evidence? BMJ. 2020;370:m3166.

21. Al-Jabir A, Kerwan A, Nicola M, Alsafi Z, Khan M, Sohrabi $\mathrm{C}$, et al. Impact of the Coronavirus (COVID-19) pandemic on surgical practice-part 1. Int J Surg (London, England). 2020;79:168-79.

22. Iyengar KP, Jain VK, Vaish A, Vaishya R, Maini L, Lal H. Post COVID-19: planning strategies to resume orthopaedic surgerychallenges and considerations. J Clin Orthopaedics Trauma. 2020;11(Suppl 3):S291-5.

23. Office of National Statistics. Coronavirus (COVID-19) Infection Survey pilot: England, 25 June 2020 2020. https://www. ons.gov.uk/peoplepopulationandcommunity/healthandsocial care/conditionsanddiseases/bulletins/coronaviruscovid19infecti onsurveypilot/england25june2020\#number-of-people-in-engla nd-who-had-covid-19.

24. Office of National Statistics. Subnational population projections for England: 2018-based. https://www.ons.gov.uk/peoplepopulatio nandcommunity/populationandmigration/populationprojections/ bulletins/subnationalpopulationprojectionsforengland/2018based\# change-by-region. 